\title{
Organização dos serviços públicos de saúde bucal para diagnóstico precoce de desordens com potencial de malignização do estado do Rio de Janeiro, Brasil
}

\author{
Organization of public oral health services for early diagnosis of \\ potentially malignant disorders in the state of Rio de Janeiro, Brazil
}

Elisete Casotti ${ }^{1}$

Ana Beatriz Fonseca Monteiro ${ }^{2}$

Evelyn Lima de Castro Filho ${ }^{3}$

Manuella Pires dos Santos ${ }^{3}$

${ }^{1}$ Instituto de Saúde Coletiva,

Universidade Federal

Fluminense (UFF). R.

Marquês de Paraná 303/

Prédio Anexo $/ 3^{\circ}$, Centro.

24033-900. Niterói RJ Brasil.

elisete.casotti@gmail.com

${ }^{2}$ Instituto de Matemática e

Estatística, UFF. Niterói RJ

Brasil.

${ }^{3}$ Faculdade de Odontologia,

UFF. Niterói RJ Brasil.

\begin{abstract}
This is a study of the organization of public health services in the state of Rio de Janeiro concerning the diagnosis of potentially malignant disorders. Secondary data from the database of the first phase of the Program for Enhancement for Access to and Quality of Primary Care were used. The implementation of actions at different levels for cancer prevention, the availability of diagnostic support services and the organization of the care network were assessed. The results show that only $58.8 \%$ of oral health teams record and monitor suspect cases; that only $47.1 \%$ reported having preferential channels for referring patients and there is great variation in waiting times to confirm the diagnosis. Local managerial and regional support actions can improve the organization of the care network for oral cancer prevention in the state.
\end{abstract}

Key words Primary Health Care, Evaluation of health services, Dentistry in public health, Oral health, Oral neoplasms
Resumo Estudo sobre a organização dos serviços públicos de saúde no estado do Rio de Janeiro referente ao diagnóstico precoce de desordens com potencial de malignização (DPM). Foram utilizados os dados secundários do banco do primeiro ciclo do Programa de Melhoria do Acesso e Qualidade da Atenção Básica.Verificou-se a realização de ações nos diferentes níveis de prevenção ao câncer, a disponibilidade de serviços de apoio diagnóstico e sobre a organização da rede de atenção. Os resultados mostram que só $58,8 \%$ das equipes de saúde bucal registram e acompanham os casos suspeitos; que somente $47.1 \%$ relatam dispor de fluxos preferenciais para encaminhamento e há grande variação nos tempos de espera para confirmação do diagnóstico. Ações gerenciais locais e de apoio regional podem melhorar a organização da rede de cuidado ao câncer bucal no estado.

Palavras-chave Atenção Primária à Saúde, Avaliação de serviços de saúde, Odontologia em saúde pública, Saúde bucal, Neoplasias bucais 


\section{Introdução}

É considerado câncer de boca as neoplasias que afetam os lábios e o interior da cavidade oral (gengivas, mucosa jugal, palato duro, língua e assoalho bucal). Histologicamente, o carcinoma de células escamosas é omais frequente, respondendo por cerca de 90 a $95 \%$ dos $\operatorname{casos}^{1,2}$.

A incidência de câncer de boca é um problema de saúde pública no mundo, com taxa anual estimada em aproximadamente 275 mil casos, sendo dois terços destes em países em desenvolvimento $^{1,3}$. No Brasil, a estimativa para o ano de 2014 era de 11.280 casos novos de câncer da cavidade oral em homens e 4.010 em mulheres ${ }^{4}$.

Ainda que avanços no diagnóstico e tratamentode várias formas de tumores malignostenham resultado no aumento de sobrevidados pacientes, os indicadores epidemiológicos do câncer da boca não têm apresentado melhora ${ }^{5,6}$. A natureza silenciosa das lesões e o atraso no diagnóstico são fatores relacionados com a identificação tardia e, consequentemente, com o estadiamento avançado das lesões ${ }^{7,8}$.

$\mathrm{O}$ atraso do diagnóstico pode estar associado ao tempo em que o paciente leva para perceber o seu adoecimento e procurar auxílio profissional (evolução inicial oligossintomática da doença); às dificuldades de acesso aos serviços de saúde bucal'; e àfalta de informações associada à maior vulnerabilidade social do grupo de risco (homens, maiores de 50 anos, etilistas e fumantes, baixo estrato socioeconômico e educacional $)^{6,10,11}$.

$\mathrm{O}$ diagnóstico precoce de câncer bucal e o imediato encaminhamento do paciente para tratamento são fatores importantes para a redução da morbidade e mortalidade causadas pela doen$c_{c}{ }^{12}$. Nos casos de diagnóstico precoce, as complicações no tratamento podem ser minimizadas, as intervenções podem ser mais conservadoras (não mutiladoras) e os resultados estéticos e funcionais melhores, aumentando o índice de sobrevida e de qualidade de vida do paciente ${ }^{13}$.

Segundo o estudo de Reis et al. ${ }^{14}$, a chance de sobrevida para pacientes em estágio avançado é de $50 \%$ ou menos para um período de cinco anos, porém, se a lesão for diagnosticada ainda pequena e localizada, taxas de $70 \%$ a $90 \%$ podem ser atingidas. Contudo, numerosos estudos sugerem que até $50 \%$ dos pacientes apresentam a doença em estágio avançado no momento do diagnóstico ${ }^{15-17}$.

Brocklehurstet al. ${ }^{18}$ apontam que ainda não há evidência de que um exame visual, como parte de um programa de rastreamento de base popu- lacional, reduza a taxa de mortalidade por câncer bucal. Entretanto, autores têm defendido que estratégias de identificação de casos em indivíduos com os principais fatores de risco poderiam resultar em uma maior chance de prevenção secundária ${ }^{19-21}$.

Desde a aprovação da Política Nacional de Saúde Bucal, em 2004, a identificação precoce de lesões de boca, o acompanhamento dos casos suspeitos e a garantia de tratamento e reabilitação, mediante articulação em rede, são ações identificadas como necessárias para a ampliação e a qualificação da atenção básica no Sistema Único de Saúde ${ }^{22}$.

A recente ampliação do acesso aos serviços odontológicos no país, seja na atenção primária com a expansão das equipes de saúde bucal (ESB) ou da média complexidade com os Centros de Especialidades Odontológicas (CEO), ainda não significou o desenvolvimento e a implantação de tecnologias destinadas a monitorar os determinantes, os riscos e os danos referidos ao câncer de boca. Avanços na direção da consolidação de uma política de vigilância em saúde bucal no Brasil têm sido reportados por alguns autores ${ }^{23,24}$, mas as iniciativas envolvem principalmente os inquéritos populacionais das doenças bucais mais prevalentes e a inclusão de módulos temáticos em pesquisas com grupos específicos, como na Vigilância de Fatores de Risco e Proteção para Doenças Crônicas por Inquérito Telefônico (VIGITEL), noInquérito Nacional sobre Violências e Acidentes em Serviços Sentinela de Urgência e Emergência (VIVA) e na Pesquisa Nacional de Saúde do Escolar (PeNSE).

Entretanto, não há registro de que esteja em curso alguma estratégia específica para o enfrentamento da situação epidemiológica do câncer bucal. Torres-Pereira ${ }^{25}$, analisando criticamente as políticas públicas para o câncer de boca de âmbito nacional, conclui que há "uma preocupação mais institucional por conta do trabalho quase isolado de profissionais ou de associações dos mesmos e menos uma atenção de cunho governamental".

$\mathrm{Na}$ tentativa de induzirmudanças qualitativas no sistema de saúde brasileiro e estabelecer uma cultura avaliativa, o Ministério da Saúde instituiu em 2012 o Programa Nacional de Melhoria do Acesso e da Qualidade da Atenção Básica/PMA$\mathrm{Q}-\mathrm{AB}^{26}$. A problemática do câncer bucal foi incluída diretamente na seção sobre a organização da atenção em saúde bucal e indiretamente em outras duas seções, permitindo um estudo exploratório sobreo tema. 


\section{Metodologia}

Trata-se de um estudo descritivo, que utilizou dados secundários do instrumento denominado "Módulo II - Entrevista com Profissional da Equipe de Atenção Básica e Verificação de Documentos na Unidade de Saúde", pertencente ao banco do PMAQ_AB. Foram selecionadas sete questões da seção "Atenção à Saúde Bucal", uma referida ao "Programa Saúde na Escola" e outra da "Promoção da Saúde", elencadas nas Tabelas 1,2 e 3 . O estudo foi submetido e aprovado pelo Comitê de Ética em Pesquisas em Seres Humanos da Ensp/Fiocruz.

Os registros foram coletados durante a Avaliação Externa e incluem o universo de equipes de saúde bucal (ESB) do Estado do Rio de Janeiro que participaram do I Ciclo do Programa.Asentrevistas foram realizadas in loco e os respondentes foram o cirurgião dentista, para a seção específica, e um membro designado pela Equipe de Saúde da Família, para as demais seções.

A agregação das questões é apresentada em dois blocos: um relacionado às ações realizadas pela ESF/ESB e outro à organização da rede de serviços de apoio à atenção primária à saúde (APS).

O primeiro bloco se baseou nos níveis de prevenção das doenças, ajustado para o câncer por Torres-Pereira ${ }^{25}$, em que a prevenção primária está relacionada às ações para o controle ou eliminação dos fatores de risco (tabaco, álcool e a exposição ao sol) evitando o início da doença; a secundária referida ao diagnóstico precoce das lesões,aqui modificado, incluindo desde as não identificadas pelo paciente, até aquelascom evolução clínica aparente; e a terciária, que inclui alimitação do dano e a oferta de reabilitação às condições irremediáveis resultantes da doença.

Como o instrumento não coletou dados específicos sobre a atenção terciária, muito provavelmente porque as ações não são competência direta da APS, todas as questões ficaram alocadasnos dois primeiros níveis (Tabela 1).

$\mathrm{O}$ segundo bloco, referente à organização da rede de serviços de apoio à APS, foi subdivido

em Disponibilidade de serviços e Organização da rede, em que o primeiro descreve a presença de unidade e especialista de referência para o diagnóstico do câncer de boca, e o segundo a existência de protocolos que definem fluxos e tempo de espera (Tabela 2).

As análises foram realizadas considerando as nove regiões de saúde do Estado, mais a Capital. Os dados da Capital foram tratados separadamente da região Metropolitana I, como forma de visualizar as distintas realidades. A diferença entre os tempos médios de espera para atendimento nas regiões geográficas do Estado foi verificada através do teste não paramétrico de Kruskal-Wallis e as comparações múltiplas foram realizadas através da aplicação do teste de Mann -Whitney. Foi adotado um nível de significância global de 5\%. Para as análises, foram utilizados os softwares SPSS ${ }^{\circledR} 18.0$ e Excel ${ }^{\circledR} 2010$.

\section{Resultados e Discussão}

Do total de 92 municípios do Estado, 72 aderiram ao I ciclo do PMAQ_AB. Foi avaliado um total de 886 equipes de Saúde da Família, destas, 605 (69,85\%) tinham saúde bucal. A distribuição percentual das equipes de saúde bucal participantes, em relação ao total por região, pode ser vista no Gráfico 1.

\section{Ações de prevenção primária do câncer de boca}

A estratégia Saúde da Família (ESF), ao eleger o território como eixo central da organização do seu processo de trabalho, estabeleceuma estreita relação com a população daquele local, de modo a conheceros indivíduos, as famílias e a dinâmica da coletividade. Ao mesmo tempo em que é uma importante porta de entrada para o sistema, torna-se também o espaço privilegiado para a realização de ações de promoção da saúde e de prevenção de doenças e agravos à saúde.O uso de ferramentas como o mapeamento do território, o cadastramento das famílias e a rotina de visitas domiciliares realizadas pelos agentes comunitários de saúde, permitem reunir informações valiosas para o diagnóstico e o planejamento de ações na área ${ }^{27,28}$.

Monken e Barcellos ${ }^{27}$ defendem a ideia de que a prática de vigilância em saúde está imbricada com a organização do processo de trabalho em territórios delimitados, pois é na combinação do diagnóstico local e do uso de distintas tecnologias para o controle dos determinantes, dos riscos 
Tabela 1. Distribuição das equipes participantes do PMAQ AB, segundo as ações associadas ao cuidado com o câncer bucal. Estado do Rio de Janeiro e Regiões de Saúde, 2012.

\begin{tabular}{|c|c|c|c|c|c|c|c|}
\hline \multirow[b]{2}{*}{ Ações } & & \multirow[b]{2}{*}{ Estado } & \multicolumn{5}{|c|}{ Região de Saúde } \\
\hline & & & $\begin{array}{l}\text { B. Ilha } \\
\text { Grande }\end{array}$ & $\begin{array}{c}\text { B. } \\
\text { Litorânea }\end{array}$ & $\begin{array}{l}\text { Centro } \\
\text { a Sul }\end{array}$ & $\begin{array}{l}\text { Médio } \\
\text { Paraíba }\end{array}$ & Capital \\
\hline Prevenção primária & $\mathrm{N}$ & 605 & 17 & 41 & 73 & 105 & 190 \\
\hline $\begin{array}{l}\text { Quais as atividades de promoção e prevenção } \\
\text { que a equipe realiza? Ações de prevenção do } \\
\text { uso de álcool, tabaco e outras drogas? }\end{array}$ & $\%$ & 47,8 & 17,6 & 21,4 & 51,4 & 63,8 & 52,1 \\
\hline $\begin{array}{l}\text { A equipe oferta ações educativas e de } \\
\text { promoção da saúde direcionadas para: } \\
\text { Prevenção e tratamento ao uso, abuso e } \\
\text { dependência decorrentes do uso de crack, } \\
\text { álcool e outras drogas? }\end{array}$ & $\%$ & 22,6 & 0 & 4,8 & 13,5 & 33,3 & 25,8 \\
\hline Prevenção secundária & $\mathrm{N}$ & 605 & 17 & 41 & 73 & 105 & 190 \\
\hline $\begin{array}{l}\text { A equipe realiza campanhas para detecção de } \\
\text { lesões bucais e encaminha casos suspeitos de } \\
\text { câncer de boca? }\end{array}$ & $\%$ & 77,4 & 47,1 & 57,1 & 75,7 & 85,7 & 80 \\
\hline \multirow{2}{*}{$\begin{array}{l}\text { A equipe registra e acompanha os casos } \\
\text { suspeitos/confirmados de câncer de boca? }\end{array}$} & $\%$ & 63,8 & 47,1 & 46,6 & 45,9 & 81,9 & 70 \\
\hline & $\mathrm{N}$ & 386 & 8 & 19 & 34 & 86 & 133 \\
\hline \multirow[t]{2}{*}{ Existe documento que comprove? } & $\%$ & 58,8 & 50 & 38,2 & 62,8 & 69,2 & 55,6 \\
\hline & & & \multicolumn{5}{|c|}{ Região de Saúde } \\
\hline Ações & & Estado & $\begin{array}{c}\text { Metro } \\
\text { I }\end{array}$ & $\begin{array}{l}\text { Metro } N \\
\text { II }\end{array}$ & Noroeste & Norte & Serrana \\
\hline Prevenção primária & $\mathrm{N}$ & 605 & 31 & 32 & 37 & 38 & 41 \\
\hline $\begin{array}{l}\text { Quais as atividades de promoção e prevenção } \\
\text { que a equipe realiza? Ações de prevenção do } \\
\text { uso de álcool, tabaco e outras drogas? }\end{array}$ & $\%$ & 47,8 & 44,8 & 37,3 & 27,3 & 27,8 & 58,7 \\
\hline $\begin{array}{l}\text { A equipe oferta ações educativas e de } \\
\text { promoção da saúde direcionadas para: } \\
\text { Prevenção e tratamento ao uso, abuso e } \\
\text { dependência decorrentes do uso de crack, } \\
\text { álcool e outras drogas? }\end{array}$ & $\%$ & 22,6 & 24,1 & 29,4 & 9,1 & 16,7 & 28,3 \\
\hline Prevenção secundária & $\mathrm{N}$ & 605 & 31 & 32 & 37 & 38 & 41 \\
\hline $\begin{array}{l}\text { A equipe realiza campanhas para detecção de } \\
\text { lesões bucais e encaminha casos suspeitos de } \\
\text { câncer de boca? }\end{array}$ & $\%$ & 77,4 & 65,5 & 90,2 & 66,7 & 77,8 & 80,4 \\
\hline \multirow{2}{*}{$\begin{array}{l}\text { A equipe registra e acompanha os casos } \\
\text { suspeitos/confirmados de câncer de boca? }\end{array}$} & $\%$ & 63,8 & 62,1 & 60,8 & 54,5 & 50 & 63 \\
\hline & $\mathrm{N}$ & 386 & 20 & 22 & 21 & 18 & 25 \\
\hline Existe documento que comprove?* & $\%$ & 58,8 & 51,6 & 33,3 & 77,8 & 51,7 & 50 \\
\hline
\end{tabular}

Fonte: MS-DAB. PMAQ-AB, 2012.

* Refere-se ao total de equipes que responderam SIM a pergunta sobre o acompanhamento dos casos.

e dos danos à saúde, que ações oportunas podem ser desenvolvidas.

Analisando as medidas de prevenção primária, associadas à informação sobre os principais fatores de risco de aparecimento do câncer, 47,8\% das equipes de Saúde da Família afirmam realizar ações de prevenção do uso de álcool, ta- baco e outras drogas com escolares. Entretanto, somente $22,6 \%$ reportam desenvolver atividades de prevenção e tratamento ao uso, abuso e dependência decorrentes do uso de crack, álcool e outras drogas no território.

Segundo inquérito realizado pelo o Instituto Brasileiro de Geografia e Estatística (IBGE), em 
Tabela 2. Distribuição das equipes participantes do PMAQ AB,segundo as respostas sobre as características da rede de serviços complementar a atenção básica. Estado do Rio de Janeiro e Regiões de Saúde, 2012.

\begin{tabular}{|c|c|c|c|c|c|c|c|}
\hline \multirow[b]{2}{*}{ Características da rede } & & \multirow[b]{2}{*}{ Estado } & \multicolumn{5}{|c|}{ Região de Saúde } \\
\hline & & & $\begin{array}{l}\text { B. Ilha } \\
\text { Grande }\end{array}$ & $\begin{array}{c}\text { B. } \\
\text { Litorânea }\end{array}$ & $\begin{array}{l}\text { Centro } \\
\text { a Sul }\end{array}$ & $\begin{array}{l}\text { Médio } \\
\text { Paraíba }\end{array}$ & Capital \\
\hline Disponibilidade de serviço & $\mathrm{N}$ & 605 & 17 & 41 & 73 & 105 & 190 \\
\hline $\begin{array}{l}\text { Existe Centro Especializado de Odontologia } \\
\text { CEO de referência para a sua equipe? }\end{array}$ & $\%$ & 82,8 & 88,2 & 61,0 & 85,1 & 92,4 & 88,9 \\
\hline \multirow{2}{*}{$\begin{array}{l}\text { O município possui referência para } \\
\text { Estomatologista (CEO ou e/ou outro ponto } \\
\text { da Rede)? }\end{array}$} & $\%$ & 66,6 & 70,6 & 52,4 & 35,1 & 83,8 & 80 \\
\hline & $\mathrm{N}$ & $501^{*}$ & 15 & 25 & 62 & 97 & 169 \\
\hline $\begin{array}{l}\text { Estomatologista somente para ESB que } \\
\text { responderam ter CEO de referência }\end{array}$ & $\%$ & 74,1 & 73,3 & 57,7 & 36,5 & 77,3 & 98,8 \\
\hline Organização da rede & $\mathrm{N}$ & 605 & 17 & 41 & 73 & 105 & 190 \\
\hline \multirow{2}{*}{$\begin{array}{l}\text { Fluxos preferenciais para usuários com } \\
\text { suspeita de câncer de boca? }\end{array}$} & $\%$ & 47,1 & 16,7 & 31,1 & 90,5 & 74,7 & 58,6 \\
\hline & & & \multicolumn{5}{|c|}{ Região de Saúde } \\
\hline Características da rede & & Estado & $\begin{array}{l}\text { Metro } \\
\text { I }\end{array}$ & $\begin{array}{c}\text { Metro } \mathrm{N} \\
\text { II }\end{array}$ & Noroeste & Norte & Serrana \\
\hline Disponibilidade de serviço & $\mathrm{N}$ & 605 & 31 & 32 & 37 & 38 & 41 \\
\hline $\begin{array}{l}\text { Existe Centro Especializado de Odontologia } \\
\text { CEO de referência para a sua equipe? }\end{array}$ & $\%$ & 82,8 & 90,3 & 65,6 & 51,4 & 86,8 & 78,0 \\
\hline O município possui referência para & $\%$ & 66,6 & 82,8 & 74,5 & 36,4 & 55,6 & 41,3 \\
\hline \multicolumn{8}{|l|}{$\begin{array}{l}\text { Estomatologista (CEO ou e/ou outro ponto } \\
\text { da Rede)? }\end{array}$} \\
\hline & $\mathrm{N}$ & $501^{*}$ & 28 & 21 & 19 & 33 & 32 \\
\hline $\begin{array}{l}\text { Estomatologista somente para ESB que } \\
\text { responderam ter CEO de referência }\end{array}$ & $\%$ & 74,1 & 50,0 & 81,6 & 45 & 80 & 46,7 \\
\hline Organização da rede & $\mathrm{N}$ & 605 & 31 & 32 & 37 & 38 & 41 \\
\hline $\begin{array}{l}\text { Fluxos preferenciais para usuários com } \\
\text { suspeita de câncer de boca? }\end{array}$ & $\%$ & 47,1 & 60,8 & 33,3 & 38,9 & 34,8 & 47,1 \\
\hline
\end{tabular}

* Refere-se ao total de equipes que responderam que SIM para CEO de referência.

Fonte: MS-DAB. PMAQ-AB, 2012.

conjunto com o Instituto Nacional do Câncer $(\mathrm{INCA})^{29}$, as mais altas prevalências de tabagismo encontram-se no Sudeste e Sul, também as duas regiões com maiores incidências de neoplasias estritamente relacionadas ao tabaco (cavidade oral, esôfago e pulmão).

Apresença de grupos antitabagismo nas ESF aliada a campanhas sobre o tema nas escolas compreendem excelentes estratégias para captação, educação e motivação ao tratamento dos principais grupos de risco para o câncer tabacodependente: tabagistas crônicos na faixa etária de 45-70 anos e adolescentes ${ }^{30}$. O primeiro, por tratar-se de indivíduos oriundos de uma época na qual o estímulo ao uso de tabaco superava o esclarecimento de seus males; cujo consumo prolongado lhes dificulta a pausa e cuja curva de mortalidade por câncer de pulmão ainda se encontra ascendente ${ }^{31}$. O segundo, por tratar-se de indivíduos que devem ser protegidos, uma vez que, quanto mais precoce inicia-se o consumo de tabaco, maior a probabilidade de o indivíduo tornar-se dependente da nicotina e maior o risco de desenvolvimento de algum tipo de câncer relacionado a esse uso na vida adulta produtiva ${ }^{32}$.

\section{Prevenção secundária do câncer de boca}

A principal medida de prevenção secundária para o câncer bucal éa realização, o mais preco- 
Tabela 3. Distribuição das equipes participantes do PMAQ, segundo o tempo de espera relatado para agendamento de consulta com o estomatologista. Estado do Rio de Janeiro e Regiões de Saúde, 2012.

\begin{tabular}{|c|c|c|c|c|c|c|}
\hline \multirow[b]{2}{*}{ Região } & \multirow[b]{2}{*}{ Média } & \multirow[b]{2}{*}{$\begin{array}{l}\text { Desvio } \\
\text { padrão }\end{array}$} & \multirow[b]{2}{*}{ Mínimo } & \multicolumn{3}{|c|}{ Tempo de espera } \\
\hline & & & & Máximo & Mediana & $\begin{array}{l}\text { Equipes que conseguem } \\
\text { vaga em até } 20 \text { dias (\%) }\end{array}$ \\
\hline Baía da Ilha Grande & 48,42 & 43,92 & 7 & 99 & 30 & $41,7(\mathrm{n}=12)$ \\
\hline Baixada Litorânea & 21,91 & 22,24 & 1 & 99 & 15 & $72,7(\mathrm{n}=22)$ \\
\hline Centro Sul & 12,42 & 10,60 & 1 & 30 & 7 & $76,9(\mathrm{n}=26)$ \\
\hline Médio Paraíba & 23,73 & 31,32 & 1 & 99 & 15 & $75,0(\mathrm{n}=88)$ \\
\hline Metropolitana I - Só Capital & 14,07 & 18,90 & 1 & 120 & 7 & $80,9(\mathrm{n}=152)$ \\
\hline Metropolitana I - demais (s/ RJ) & 50,46 & 48,18 & 1 & 180 & 30 & $45,8(\mathrm{n}=24)$ \\
\hline Metropolitana II & 16,97 & 21,04 & 1 & 99 & 7 & $84,2(\mathrm{n}=38)$ \\
\hline Noroeste & 29,83 & 41,71 & 5 & 99 & 7 & $75,0(\mathrm{n}=12)$ \\
\hline Norte & 49,60 & 34,24 & 15 & 98 & 35 & $30,0(\mathrm{n}=10)$ \\
\hline Serrana & 58,58 & 41,26 & 3 & 99 & 60 & $26,3(\mathrm{n}=19)$ \\
\hline Geral & 23,41 & 30,66 & 1 & 180 & 10 & $72,0(\mathrm{n}=403)$ \\
\hline
\end{tabular}

Fonte: MS-DAB. PMAQ-AB, 2012.

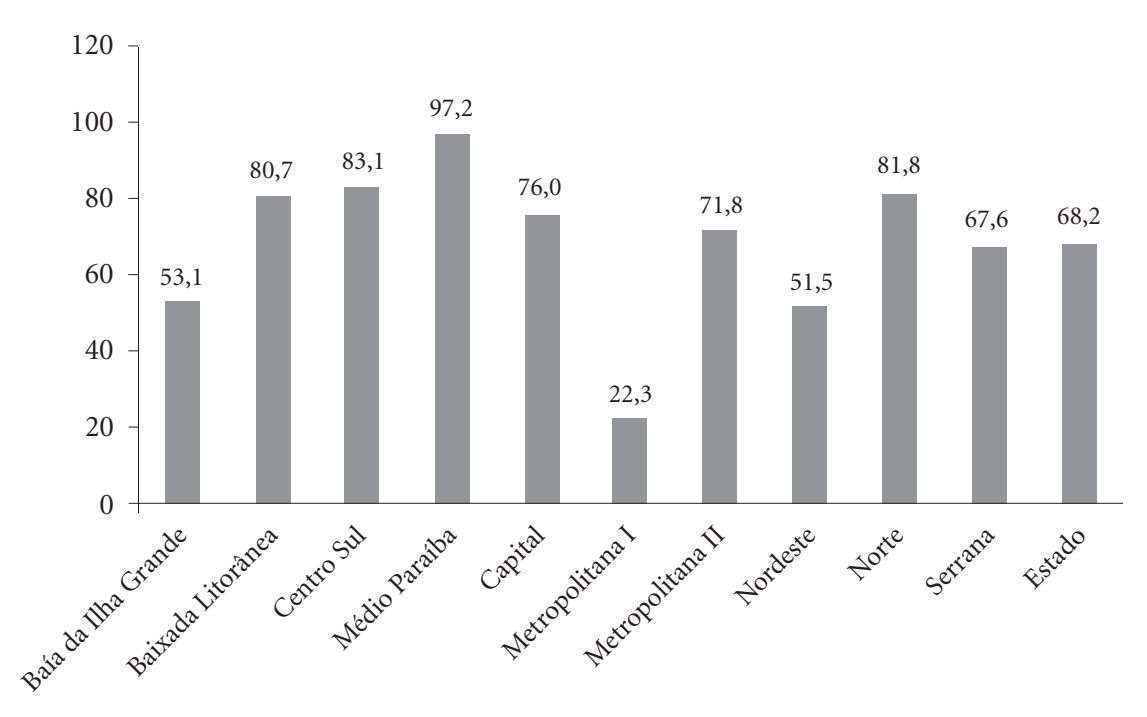

Gráfico 1. Percentual de Equipes de Saúde Bucal participantes do PMAQ-AB, segundo Regiões de Saúde. Estado do Rio de Janeiro, 2012.

Fonte: MS-DAB. PMAQ-AB, 2012.

cemente possível, do diagnóstico das desordens com potencial de malignização (DPM), seja antecipando-se à percepção do próprio paciente ou atendendo a sua queixa clínica.

Neste estudo, 77,4\% $(\mathrm{n}=468)$ das equipes de saúde bucal informaram realizar campanhas para detecção precoce e encaminhamento dos casos suspeitos, destas 63,8\% $(\mathrm{n}=386)$ responderam que registram e acompanham, mas somente um pouco mais da metade comprovou com documentos a afirmação $(n=227)$.

Regiões como Centro Sul, Médio Paraíba, Capital, Metro II, Norte e Serrana alcançaram percentuais acima de $75 \%$ de equipes que reporta- 
ram realizar campanhas e encaminhar casos suspeitos. Entretanto, as Regiões Baía da Ilha Grande, Baixada Litorânea, Metro I, Metro II, Norte e Serrana não ultrapassaram a marca de 52\% de ESB que comprovaram registrar e acompanhar os casos. Destaca-se, portanto, a marcante diferença entre o relatado e o comprovado.

A questão do registro e acompanhamento dos indivíduos portadores de DPM pela atenção primária diz respeito ao cumprimento de dois atributos essenciais da APS, quais sejam: da coordenação e da integralidade do cuidado. Para Franco e Franco ${ }^{33}$, a responsabilidade sobre o cuidado do paciente, incluindo a gestão do projeto terapêutico é dos profissionais da atenção básica, responsabilidade que inclui o acompanhamento do itinerário do usuário aos outros níveis de assistência, assim como a todos os fluxos assistenciais, garantindo que o vínculo entre o usuário e a atenção básica seja preservado.

Da perspectiva clínica, com a inclusão de novos fatores de risco associados ao câncer bucal, como os relacionados à imunosupressão (casos de pessoas com Aids e/ou submetidos a transplante de medula óssea $)^{34}$, e a ausência de um marcador confiável que permita prever a transformação maligna de uma DPM oral ${ }^{35,36}$, a vigilância em saúde e o acompanhamento dos casos suspeitos torna-se uma medida fundamental para o diagnóstico precoce e o pronto tratamento.

Para Torres-Pereira ${ }^{25}$, uma das questões que precisa ser considerada na atenção secundária ao câncer, face aos novos conhecimentos sobre as DPM orais e a mudança do perfil esperado do paciente, é sobre a capacitação dos profissionais de saúde. Incorporar novos conhecimentos sobre a doença e reorganizar as práticas de vigilância em saúde nos territórios são partes importantes de uma mudança necessária na atenção primária e secundária ao câncer bucal.

\section{Características da rede de atenção}

A concepção de um sistema de saúde em rede coordenada pela APS tem três componentes essenciais: uma população definida, uma estrutura operacional (serviços) e um modelo de atenção ${ }^{37}$. Aqui será analisada a estrutura disponível para a oferta de procedimentos de média complexidade e a existência de fluxos organizadores entre a APS e esse nível de atenção.

\section{Disponibilidade de serviço de média complexidade}

Os Centros de Especialidades Odontológicas são as estruturas criadas para responder pelas ações de saúde bucal de nível secundário complementares à APS, que atuam dando cobertura para um número de ESB definidas de acordo com um plano de regionalização. Segundo a Portaria MS/GM 599 de 23 de março de 2006, o diagnóstico oral é uma das cinco especialidades previstas para os CEO sem, contudo, haver exigência de habilitação específica do profissional que a executa. As diretrizes de funcionamento constam do Manual de Especialidades em Saúde Bucal ${ }^{38,39}$.

No Estado do Rio de Janeiro, no ano da coleta dos dados, estavam emfuncionamento $65 \mathrm{CEO}$, sendo a região Norte a única que não tinha Centro implantado ${ }^{40}$.

O diagnóstico, envolvendo a biópsia e a citologia esfoliativa, assim como os demais exames complementares, é facultativo aos dentistas da APS, desde que se sintam capacitados para a coleta, o pedido e a interpretação dos resultados. Entretanto, nos casos de encaminhamento, o Manual ressalta "que é de extrema importância o acompanhamento pelos profissionais da UBS/ SF dos casos encaminhados aos outros níveis de atenção, na perspectiva da continuidade do cuidado" $^{38}$.

No Estado, 82,8\% das ESB responderam ter CEO de referência. Quando perguntados sobre a existência de referência para a especialidade deestomatologia, $66 \%$ responderam positivamente. Analisando apenas o grupo daquelas com CEO de referência, somente $74,1 \%$ responderam positivamente para acesso à especialidade de estomatologia. Ou seja, aparentemente, de cada 10 CEO em funcionamento no Estado, 3 não oferecem o serviço de diagnóstico oral.

A região Baixada Litorânea e a Noroeste são as que apresentam as maiores dificuldades de acesso à rede de média complexidade em saúde bucal. Entretanto, destaca-se a organização da rede que apóia os municípios da região Norte, onde mesmo não sediando nenhum Centro 86,8\% das ESB referem disponibilidade do serviço.

A presença de unidades de referência com especialistas qualificados para o diagnóstico dos casos encaminhados é condição sinequa non para apoiar e qualificar o trabalho das equipes de atenção básica. 


\section{Organização do funcionamento da Rede de Atenção}

A criação de fluxos assistenciais são estratégias que organizam o funcionamento das redes de atenção à saúde, permitindo que as equipes de APS possam interagir com os demais serviços de acordo com as necessidades dos usuários sob seus cuidados. É de responsabilidade dos Estados, dentre outras atribuições, a coordenação da organização e da implantação dos planos regionais de atenção, nos quais está incluída a Rede de Atenção à Saúde das Pessoas com Doenças Crônicas no âmbito do SUS, bem como apoiar apactuação e a regulação das vagas e monitorar os fluxos entre os diferentes pontos de atenção ${ }^{41}$.

Neste estudo, a disponibilidade de referência dos CEO para a grande maioria das ESB não está relacionada diretamente com a existência de fluxos preferenciais para usuários com suspeita de câncer de boca $(47,1 \%)$. Em seis das nove regiões do Estado, menos da metade de ESB respondentes reconheceram dispor de algum fluxo preferencial.

O desconhecimento de fluxos pelas ESBque orientem a utilização dos serviços nos demais níveis de atenção demonstraa fragmentação destes na rede de saúde bucal. As consequências envolvem desde o deslocamento desnecessário do usuário entre os pontos de atenção, o não reconhecimento das diferentes necessidades e da urgência de algunsatendimentos até a demonstração do isolamento e incomunicabilidade entre os serviços que deveriam funcionar organizados em uma rede de apoio loco-regional.

Em geral, 72\% das ESBs relataram um tempo de espera de até 20 dias para o atendimento por um estomatologista. Tomando como base esse limite, a comparação dos desempenhos entre as regiões mostrou que mais de $76 \%$ das ESB da Metropolitana II, da Capital e da Centro-Sulconseguem atendimento dentro desse prazo. Ficando o pior desempenho com as regiões Serrana, Norte e Baía da Ilha Grande, onde menos de 42\% das ESB conseguem tal nível de adequação (Tabela 3).

Foi observada uma grande variação entre as equipes quanto aos tempos mínimos e máximos de espera para atendimento com o estomatologista. $\mathrm{Na}$ Capital, enquanto uma equipe relata que os pacientes aguardam, em média, 1 (um) dia para serem atendidos por outra equipe da mesma região, o tempo médio é de 120 dias. A melhor situação acontece dentro da região Centro-Sul, onde os tempos de espera mínimo e máximo fo- ram, respectivamente, de 1 e de 30 dias (Tabela 3). Foi encontrada diferença significativa entre as regiões quanto ao tempo médio de atendimento pelo estomatologista ( $\mathrm{p}<0,001)$. As comparações múltiplas realizadas entre os tempos de espera, entre as regiões, apenas corroboram as diferenças de padrão de atendimento observadas, para melhor, na região Centro Sul e na Capital, e para pior, nas seguintes regiões: Metropolitana I (sem a Capital), Norte e Serrana.

A grande variação entre os tempos mínimos e máximos relatados pelas ESB sugere uma fraca articulação entre os serviços de diferentes complexidades, sejam eles locais ou regionais. A ausência de fluxos conhecidos e pactuados para o câncer bucal aumenta a vulnerabilidade dos usuários e fragiliza a atuação das equipes.Identificar os nós críticos e ampliar a discussão sobre o acesso a serviços de diagnóstico (clínicos e laboratoriais) parece ser uma necessidade urgente que deve ser assumida pela gestão nas diferentes esferas governamentais.

O tempo de espera para o tratamento, etapa a seguir para os casos confirmados, não foi objeto de pesquisa deste instrumento. Entretanto, essa é uma informação importante, que deverá ser objeto de outros estudos, pois ao indicar a capacidade da rede de atenção oncológica absorver os casos, mostrará o tempo real que um paciente aguarda entre o diagnóstico (precoce ou tardio) e a entrada no processo terapêutico.

\section{Considerações Finais}

Ainda que os resultados aqui apresentados estejam referidos somente às equipes que participaram do PMAQ-AB no Estado do Rio de Janeiro, que seja considerada a influência do processo de certificação do trabalho das equipes na constituição do banco de dados e o limite explicativo de dados exclusivamente quantitativos - face à complexidade e variabilidade das modelagens assistenciais que operam simultaneamente nos municípios - ainda assim há indicações importantes sobre a insuficiência da rede de saúde bucal na atenção aos casos de câncer.

Constituir um modelo de vigilância que reorganize as ações no território, qualificar e atualizaros dentistas da estratégia Saúde da Família, construir fluxos preferenciais para o encaminhamento dos casos/suspeitos e garantir a obtenção de diagnóstico em tempo oportuno, são questões atuais e importantes que precisam ser enfrentadas pela gestão. Entretanto, são tarefas que devem 
ser construídas envolvendo profissionais dos diferentes pontos de atenção, pois o resultado final depende de uma atuação cooperativa e em rede.

\section{Colaboradores}

E Casotti trabalhou na concepção e na redação final do manuscrito; ABF Monteiro trabalhou no tratamento e na análise dos dados e na revisão final do manuscrito; EL Castro Filho e MPSantos contribuíram nas seguintes etapas: de revisão da literatura; redação da introdução e discussão dos achados.

\section{Referências}

1. Instituto Nacional de Câncer José Alencar Gomes da Silva. Tipos de Câncer. 2014. [acessado 2014 mar 2]. Disponível em: http://www2.inca.gov.br/wps/wcm/connect /tiposdecancer/site/home/boca

2. Worrall SF. Oral Cancer. [acessado 2003 abr 26]. Disponível em: http://millennium3.org.uk.

3. Warnakulasuriya S. Global Epidemiology of oral and oropharyngeal câncer. Oral Oncol 2009; 45(4-5):309316.

4. Instituto Nacional de Câncer José Alencar Gomes da Silva. Estimativa 2014. Incidência de Câncer no Brasil. 2014b. [acessado 2014 mar 2]. Disponível em: http:// www.inca.gov.br/estimativa/2014/sintese-de-resultados-comentarios.asp

5. Camargo-Cancela M, Voti L, Guerra-Yi M, Chapuis F, Mazuir M, Curado MP. Oral cavity câncer in developed and in developing countries: population based incidence. Head Neck 2010; 32(3):357-367.

6. Instituto Nacional de Câncer. Estimativas de incidência do cancer no Brasil 2010. [acessado 2010 dez 30]. Disponível em: http://www.inca.gov.br/estimativa/2010/.

7. Scott SE, Grunfeld EA, McGurk M. The idiosyncratic relationship between diagnostic delay and stage of oral squamous cell carcinoma. Oral Oncol 2005; 41(4):396403.

8. Onizawa K, Nishihara K, Yamagata K, Yusa H, Yanagawa T, Yoshida H. Factors associated with diagnostic delay of oral squamous cell carcinoma. Oral Oncol 2003; 39(8):781-788.

9. Santos LCO, Batista OM, Cangussu MCT. Caracterização do diagnóstico tardio do câncer de boca no estado de Alagoas. Braz J Otorhinolaryngol 2010; 76(4):416422.

10. Kowalski ISG, Souza CP. Social representations of relatives and patients with oral and oropharyngeal squamous carcinoma on the prevention and diagnosis of cancer. Acta Oncol Bras 2001; 21(1):206-210.

11. Conway DI, Petticrew M, Marlborough H, Berthiller J, Hashibe M, MacPherson LM. Socieconomic inequalities and oral risk: a systematic review and meta-analysis of case-control studies. Int J Cancer 2008; 122(12):2811-2819.

12. Brad WN, Terry AD. Oral Cancer and Precancerous Lesions. CA Cancer J Clin 2002; 52:195-215.

13. Lima DS, França bHS, Ignácio AS, Baioni CS. Conhecimento de alunos universitários sobre câncer bucal. Rev Brasileira de Cancerologia 2005; 51(4):283-288. 
14. Reis L, Eisner M, Kosary C, Hankey B, Miller B, Clegg L, Mariotto A, Feuer EJ, Edwards BK, editors. SEER Cancer statistics review, 1973-1997. Bethsda: National Cancer Institute; 2000.

15. Scott SE, Grunfeld EA, McGurk M. Patient's delay in oral cancer: A systematic review. Community Dent Oral Epidemiol 2006; 34(5):337-343.

16. Wildt J, Bundgaard T, Bentzen SM. Delay in the diagnosis of oral squamous cell carcinoma. Clin Otolaryngol 1995; 20(1):21-25.

17. Gomez I, Warnakulasuriya S, Varela-Centelles PI, Lopez-Jornet P, Suarez-Cunqueiro M, Diz-Dios P, Seoani J. Is early diagnosis of oral cancer a feasible objective? Who is to blame for diagnostic delay? Oral Dis 2010; 16(1):333-342.

18. Brocklehurts P, Kujan O, Glenny AM, Oliver R, Sloan P, Ogden G, Shepherd S. Screening programmes for the early detection and prevention of oral cancer. Cochrane Database Syst Rev 2010; (11):CD0044150.

19. Warnakulasuriya S, Dietrich T, Bornstein MM, Casals Peidró E, Preshaw PM, Walter C, Wennström JL, Bergström J. Oral health risks of to baccouse and effects of cessation. Int Dent J 2010; 60(1):7-30.

20. Laronde DM, Bottorff JL, Hislop TG, Poh CY, Currie B, Williams PM, Rosin MP. Voices from the community experiences from dental office: initiating oral cancer screening. J Can Dent Assoc 2008; 74(3):239-241.

21. Shuman AG, Entezami P, Chernin AS, Wallace NE, Taylor JM, Hogikyan ND. Dermographics and efficacy of head and neck cancer screening. Otolaryngol Head Neck Surg 2010; 143(3):353-360.

22. Brasil. Ministério da Saúde (MS). Secretaria de Atenção à Saúde. Departamento de Atenção Básica. Diretrizes da Política Nacional de Saúde Bucal. Brasília: MS; 2004.

23. Goes PSA, Figueiredo N, Pucca JGA, Moura L. Vigilância à saúde bucal: a construção de um modelo integrado. Cad Saude Publica 2012; 28(Supl.):s6-s7.

24. Moyses SJ, Pucca JGA, Paludetto JM, Moura L. Avanços e desafios a Politica de Vigilancia a Saude Bucal no Brasil. Rev SaudePublica2013;47(Supl. 3):161-167.

25. Torres-Pereira C. Oral cancer public policies: Is there any evidence of impact? Braz Oral Res 2010;24(Spec. Iss. 1):37-42.

26. Brasil.Ministério da Saúde (MS). Secretaria de Atenção à Saúde. Departamento de Atenção Básica. Política Nacional de Atenção Básica. Brasília: MS; 2012.

27. Monken M, Barcellos C. Vigilância em saúde e território utilizado: possibilidades teóricas e metodológicas. CadSaude Publica2005;21(3):898-906.

28. Gondim GMM, Monken M, Rojas LI, Barcellos C, Peiter P, Navarro M, Gracie R. O território da saúde: a organização do sistema de saúde e a territorialização[internet]. In: Miranda AC, Barcellos C, Moreira JC, Monken M.Território, ambiente e saúde. Rio de Janeiro: Editora Fiocruz; 2008. [acessado 2015 abr 5]. Disponível em: http://www.escoladesaude.pr.gov.br/arquivos/ File/TEXTOS_CURSO_VIGILANCIA/20.pdf

29. Instituto Brasileiro de Geografia e Estatística(IBGE). Pesquisa Nacional porAmostra de Domicílios (PNAD) Tabagismo, 2008. [acessado 2009 dez 9]. Disponível em: http://www.ibge.gov.br/home/estatistica/populacao/ trabalhoerendimento/pnad2008/suplementos/tabagismo/pnad-tabagismo.pdf]
30. Wunsch Filho V, Mirra AP, Lopez RV, Mendoza e Antunes, Leopoldo F. Tabagismo e câncer no Brasil: evidências e perspectivas. Rev. Bras. Epidemiol 2010; 13(2):175-187.

31. Malta DC, Moura LM, Souza MFM, Curado MP, Alencar AP, Alencar GP. Tendência da mortalidade do câncer de pulmão, traquéia e brônquios no Brasil, 19802003. J Bras Pneumol 2007; 33(5):536-543.

32. Everett SA, Warren CW, Sharp D, Kann L, Husten CG, Crossett LS. Initiation of cigarette smoking and subsequent smoking behavior among U.S. high school students. Prev Med 1999; 29(5):327-333.

33. Franco CM, Franco TB. Linhas de Cuidado, Passo a Passo. In:Pessôa LRS, Arruda EH, Torres KRBO, organizadores. Manual do Gerente: desafios da média gerência na saúde. Rio de Janeiro: ENSP; 2011. p. 61-75.

34. Kruse AL, Grätz KW. Oral carcinoma after he-matopoietic stem cell transplantation: a new classification based on a literature review over 30 years. Head Neck Oncol 2009; 1:29.

35. Van der Waal I. Potentially malignant disorders of the oral and oropharyngeal mucosa; terminology, classification and present concepts of management. Oral Oncology 2009; 45(4-5):317-323.

36. Farah CS, Woo S,Zain RB,Klavounou A, McCullough MJ, Lingen M. Oral Cancer and Oral Potentially Malignant Disorders. Int J Dent 2014; 2014:853479.

37. Organização Panamericana de Saúde (OPAS). A atenção à saúde coordenada pela APS: construindo as redes de atenção no SUS: contribuições para o debate. Brasília: OPAS; 2011

38. Brasil. Ministério da Saúde (MS). Secretaria de Atenção à Saúde. Departamento de Atenção Básica. Manual de especialidades em saúde bucal. Brasília: MS; 2008. (Série A. Normas e Manuais Técnicos)

39. Brasil. Ministério da Saúde. Portaria no 599/GM, de 23 de março de 2006. Define a implantação de Centros de Especialidades Odontológicas (CEOs) e de Laboratórios Regionais de Próteses Dentárias (LRPDs) e estabelece critérios, normas e requisitos para seu credenciamento. Diário Oficial da União 2006; 24 mar.

40. Brasil. Ministério da Saúde (MS). Secretaria de Atenção à Saúde.e-SUS Atenção Básica. Manual do Sistema com Coleta de Dados Simplificada: CDS [recurso eletrônico]. Brasília: MS; 2014.

41. Brasil. Ministério da Saúde.Portaria no 874, de 16 de maio de 2013. Institui a Política Nacional para a Prevenção e Controle do Câncer na Rede de Atenção à Saúde das Pessoas com Doenças Crônicas no âmbito do Sistema Único de Saúde (SUS). Diário Oficial da União 2013; 17 maio.

Artigo apresentado em 18/06/2015

Aprovado em 22/08/2015

Versão final apresentada em 24/08/2015 